\title{
The determination of reasonable number of soil infiltration test in farmland
}

\author{
Xu Yang ${ }^{1}$, Jiamin $\mathrm{Yu}^{1}$, Yangren Wang ${ }^{1,2,}{ }^{*}$, Yanjie $\mathrm{Li}^{1}$ \\ ${ }^{1}$ Department of Water Conservancy Engineering, Tianjin Agricultural University, Tianjin 300384 \\ ${ }^{2}$ Tianjin Water - Saving Irrigation Technology and Equipment School - Enterprise Innovation Laboratory, Tianjin 300384
}

\begin{abstract}
At the experimental base of Tianjin Agricultural University, the infiltration process of water was measured by double - ring infiltration instrument at six points. Horton,Philip and Kostiakov-lewis (K-1) infiltration models were fitted with the infiltration data measured and the parameters were correspondingly obtained. Six sets of parameters of the corresponding infiltration model were obtained, and six sets of parameters were used for statistical analysis. The reasonable number of points of the corresponding model was obtained. Then, the statistical analysis of the cumulative infiltration amount was used to obtain the variation of the number of reasonable points in the three models with time. The results show that the imitative effect of accumulative infiltration water and time in K-1 model is the best, and the curve of reasonable test points determined by K-1 model with time is located under the other two models; The reasonable number of points determined by the parameter $\mathrm{K}$ in the $\mathrm{K}-1$ model is the least and most reasonable. In view of this, the number of reasonable points was determined by using the parameter $\mathrm{K}$ in the K-1 model.
\end{abstract}

\section{Introduction}

Soil infiltration refers to the process of water entering the soil. It is an important link in the process of mutual conversion of atmospheric precipitation, surface water, soil water and groundwater. It is used in many aspects such as recharge of groundwater, evaporation of phreatic water, water absorption of plant roots, and transpiration of crops. The actual condition in the field shows that in the same soil texture, soil properties (physical, chemical and biological properties) are not the same at the same time, and the characteristics are called the spatial variability of soil properties ${ }^{[1]}$. The study of soil spatial variability began in the 1960 s. Since the international academic community proposed the study of soil spatial variability in the 1970s, spatial variability of soil properties has beena hot research topicwhich were concerned by the scholars ${ }^{[2]}$, and a large number of soil properties have been studied abroad, especially the study of spatial variability in soil physical properties. Chinese scholars began to study the spatial variability of parameters in soil and water resources systems in the early 1980s. Considering the spatial variability of soil, this paper studies the number of reasonable infiltration test points in a certain farmland area.

\section{Materials and Methods}

\subsection{Study Area Overview}

The infiltration test was carried out at the Tianjin Agricultural University Experimental Base $\left(116^{\circ} 57^{\prime} \mathrm{E}\right.$, $39^{\circ} 08^{\prime} \mathrm{N}, 5.49 \mathrm{~m}$ above sea level). The base belongs to the warm temperate semi-humid continental monsoon climate. The total area of the test area is $1 \mathrm{hm} 2$. The groundwater level varies between 2.06 and $3.70 \mathrm{~m}$. The physical and chemical properties of the soil in the test area are shown in Table 1.

Table 1 Physical and chemical properties of soil in test area.

\begin{tabular}{cccccc}
\hline $\begin{array}{c}\text { Soil depth } \\
\text { /cm }\end{array}$ & Soil texture & $\begin{array}{c}\text { Mass fraction of organic matter. } \\
/ \%\end{array}$ & $\begin{array}{c}\text { Soil bulk density } \\
/{\mathrm{g} . \mathrm{cm}^{-3}}^{-}\end{array}$ & $\begin{array}{c}\text { Porosity } \\
/ \%\end{array}$ & $\begin{array}{c}\text { Field water capacity } \\
/ \%\end{array}$ \\
\hline $0 \sim 27$ & Light loam & 1.32 & 1.25 & 52.83 & 33.31 \\
$27 \sim 60$ & Light loam & 0.62 & 1.39 & 47.54 & 30.16 \\
$60 \sim 87$ & Medium loam & 0.75 & 1.39 & 47.54 & 30.16 \\
$87 \sim 120$ & Sand loam & 0.33 & 1.40 & 47.72 & 31.33 \\
\hline
\end{tabular}

\footnotetext{
*Corresponding Author's E-mail: wyrf@163.com
} 


\subsection{Research Methods}

\subsubsection{Test method}

The test site was selected in the corn field. The six measuring points are along a straight line from west to east, and the spacing between two adjacent measuring points was 15 meters. The test point arrangement is shown in Figure 1.

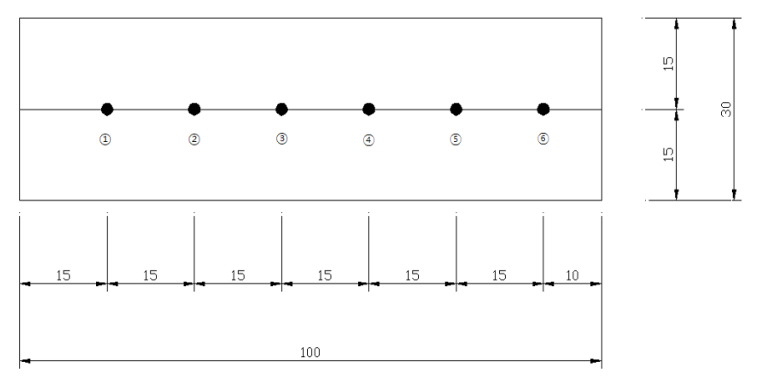

Figure 1 Layout of test points(unit:m).

The test time is May 28, 2018 (the temperature is $30^{\circ}$ C), and the six test points are numbered as(1)(2)(3)(4)(6). The infiltration process was carried out using a double-ring infiltration instrument ${ }^{[1]}$. The inner ring diameter was $40 \mathrm{~cm}$, the outer ring diameter was $60 \mathrm{~cm}$, and the inner and outer ring heights were both $25 \mathrm{~cm}$. Before the test, it was pressed against the test field and pressed into the soil depth $10 \mathrm{~cm}$. The inner ring is filled with water to maintain a head of $5 \mathrm{~cm}$. Whenever the water level in the inner ring lower $1 \mathrm{~cm}$, it is necessary to manually add water to the ring to $5 \mathrm{~cm}$ (the amount of water is $1256 \mathrm{ml}$, the amount of water is measured by the measuring cylinder) and record the amount of water poured. Add water to the outer ring so that keep the inner and outer ring water levels at the same height. After the start of the test, the amount of water in each poured and the corresponding time are recorded, and the amount of water in the inner ring is the total amount of infiltrated water. The test end until the infiltration rate approaches a constant. According to the recorded data, the infiltration rate of the six test points is basically stable after about 2 hours.

\subsubsection{Infiltration model description}

The Kostiakov-lewis(K-1), Philip and Horton infiltration formulas were chosen to fit the infiltration data at each test point. The infiltration curve was fitted using the relationship between cumulative infiltration and time. The three infiltration models are shown in Table 2.

Table 2 The three infiltration models

\begin{tabular}{|c|c|c|}
\hline Model name & Cumulative infiltration & Model parameter \\
\hline Horton & $I(t)=i_{c} t-\frac{\left(i_{0}-i_{c}\right) e^{-\beta t}}{\beta}+\frac{\left(i_{0}-i_{c}\right)}{\beta}$ & $i_{c}, i_{0}, \beta$ \\
\hline Philip & $I(t)=S t^{-0.5}+A \mathrm{t}$ & $S, A$ \\
\hline K-1 & $I(t)=k t^{\alpha}+f_{0} t$ & $k, \alpha, f_{0}$ \\
\hline
\end{tabular}

Notes: $I(t)$ is the cumulative infiltration, $\mathrm{cm} ; i_{c}$ is the stable infiltration rate; $i_{0}$ is the initial infiltration rate; $\beta$ is the empirical constant related to soil properties; $t$ is the infiltration time, $\min . S$ is the soil moisture absorption rate; $A$ is the stable infiltration rate; $K$ is infiltration coefficient; $a$ is infiltration attenuation rate; $f_{0}$ is stable infiltration rate, $\mathrm{cm} / \mathrm{min}$.

\subsubsection{Determination of reasonable measuring points}

For samples $x_{1}, x_{2}, x_{3}, \ldots \ldots, x_{i}$ of capacity $i$, the reasonable number of samples $\mathrm{N}^{[2]}$ should satisfy equation (1),

$$
P\left\{\left|\bar{x}_{i}-\mu\right| \leq \Delta\right\}=P_{1}
$$

Where ${ }^{-} x_{i}$ is the mean of the sample, $i$ is the sample point serial number, $i=1,2,3, \ldots, n, n$ is the number of samples, $\mu$ is a characteristic parameter of the normal distribution, $P_{l}$ is the confidence level, this study take $90 \%, \Delta$ is the accuracy requirement. Under the premise that the total variance value or the coefficient of variation is known, $\Delta=k \mu, k$ is $10 \%$, then $\Delta=0.1$.

However, in practice, the overall variance is unknown and can only be replaced by the variance of the sample s2. At this point, the formula (2) should be satisfied.

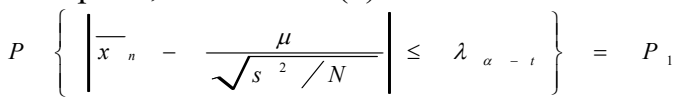

In the formula, $\lambda_{a-t}$ is the eigenvalue of the $\mathrm{t}$ distribution can be found by $\mathrm{t}$-distribution table, corresponding to the significant level $a=1-P_{l}$, the degree of freedom $f=n-1$ ( $\mathrm{n}$ is the number of measured point, $\mathrm{n}=6$ in this paper), for this study, $\lambda_{a-t}=2.015 ; \mathrm{N}$ is a reasonable number of samples. Therefore, the reasonable number of samples $\mathrm{N}$ should satisfy the formula (3).

$$
N=\lambda_{\alpha-t}^{2}\left(\frac{s}{\Delta}\right)^{2}
$$

Where, $\lambda_{a-t}$ is $4.060, \mathrm{~s}$ is standard deviation, calculated by equation (4)

$$
s=\sqrt{\frac{\sum\left(\mathrm{x}_{i}-\overline{\mathrm{x}_{i}}\right)^{2}}{n-1}}
$$

\section{Results and Analysis}

\subsection{Fitting results of model parameters}

Six test points were fitted with three models, and the fitting parameters are shown in Table 2. Taking the cumulative time as the abscissa, the total amount of infiltrated water is plotted on the ordinate, and the 
cumulative infiltration amount changes with time, as

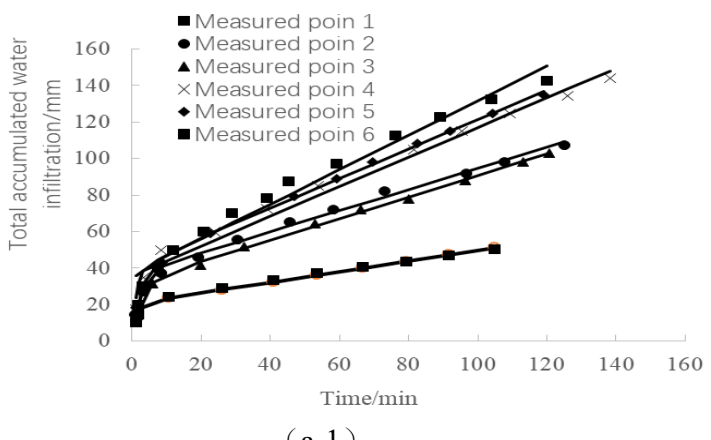

(a-1) shown in Figure 2.

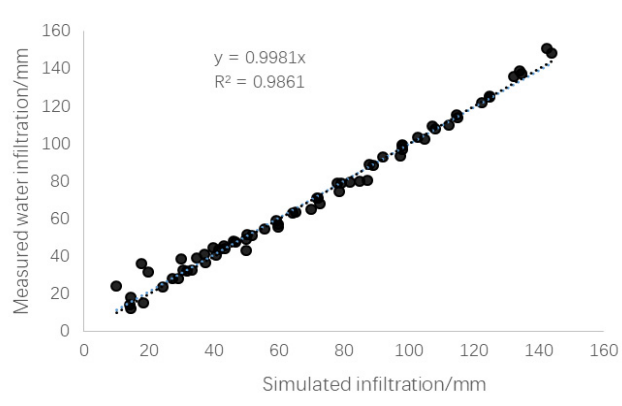

$(a-2)$

(a) Horton model.

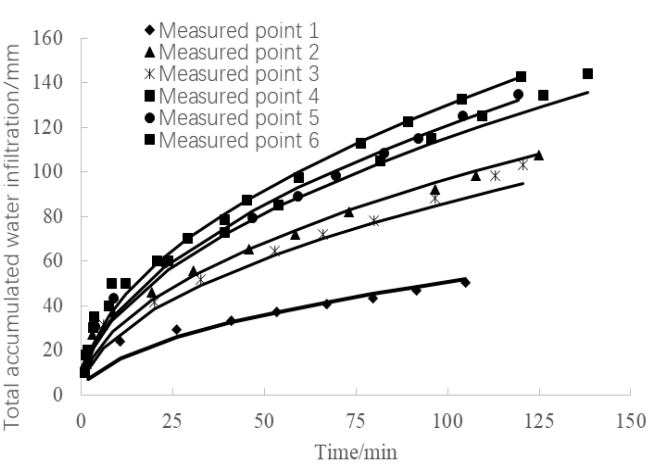

(b-1)

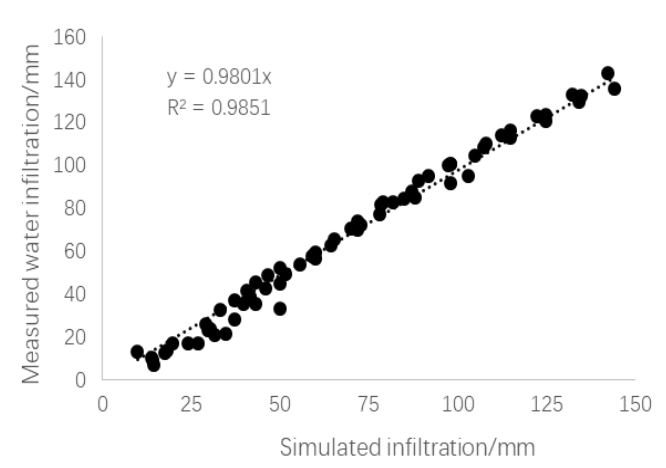

$(b-2)$

(b) Philip model.

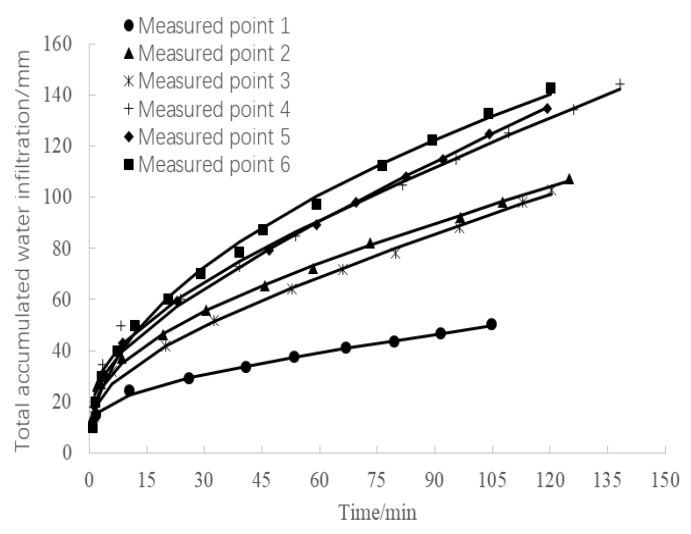

(c-1)

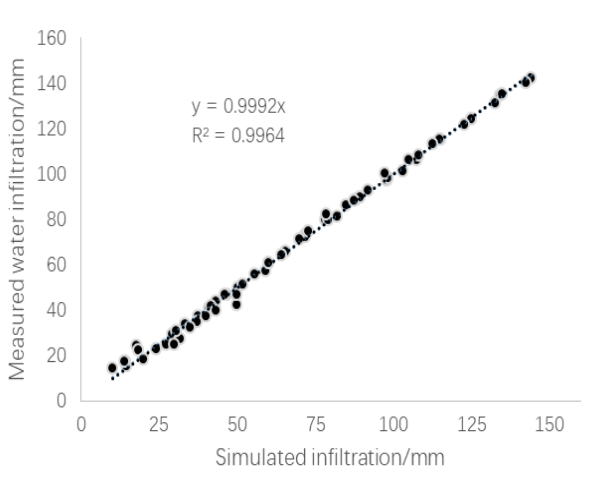

(c-2)

(c) K-1 model

Figure 2 The fitting diagram of infiltration model

It can be seen from Figure 2 that the cumulative infiltration water volume of the six measured points increases with time, the slope of the graph decreases slowly, that is, the rate of increase in total infiltration water decreases slowly with time. The cumulative infiltration water volume variation range of the six test points is $[50 \mathrm{~mm}-150 \mathrm{~mm}]$. And the cumulative infiltration water quantity is the sixth, the fifth, the fourth, the second, the third, and the first actual measurement point from the largest to the smallest under the same time condition. Figures (a-2), (b-2) and (c-2) shows the fitting of the simulated infiltration water volume with the measured infiltration water volume. Where $\mathrm{y}=\mathrm{kx}, \mathrm{k}$ is the slope, and the closer the $\mathrm{k}$ is to 1 , the better the fitting effect is; R2 is the correlation coefficient. The larger R2 is, the more accurate the model, the more significant the regression effect. Numerically, R2 is between 0-1, and the closer to 1 regression fit, the better. It can be seen from Figure 2 that the Horton model R2 $=0.9861$, the Philip model R2 $=0.9851$, the K-1 model R2 $=0.9964$. The $\mathrm{k}$ value of the K-1 model is closer to 1 than the other two models, and R2 is closer to 1, compared to the other two models. So the K-1 model fits best. The above three models fit well, indicating that the test data is reliable. In this paper, we believe that the parameters of the three models have a certain relationship with the number of reasonable points. Therefore, the parameters corresponding to the three models are used to determine 
the number of reasonable points.

\subsection{Reasonable number of measuring points}

There are 3 undetermined parameters in the Horton formula $i_{c}, i_{0}, \beta$. Six test points can get six sets of parameters accordingly. According to this, the mean and mean square error $S$ of the corresponding three pending parameters $i_{c}, i_{0}$ and $\beta$ can be obtained. Then use the formula (3) to find the corresponding reasonable number of points $\mathrm{N}$, see Table 3. Similarly, the mean, mean square error and reasonable number of points $\mathrm{N}$ of two underdetermined parameters $A$ and $S$ of the Philip formula can be obtained; The mean and mean square error of the three undetermined parameters $k$, $a$ and $f_{0}$ of the K-1 formula and the number of reasonable points $\mathrm{N}$ are also shown in Table 3.

Table 3 Table of the number of reasonable measuring points corresponding to different parameters

\begin{tabular}{ccccccccc}
\hline \multirow{2}{*}{ Test points } & \multicolumn{3}{c}{ Horton model } & \multicolumn{3}{c}{ Kostiakov-lewis } & \multicolumn{3}{c}{ Philip } \\
\cline { 2 - 9 } & $i_{c}$ & $i_{0}$ & $\beta$ & $k$ & $f_{0}$ & $\alpha$ & $S_{0}$ & $A$ \\
\hline 1 & 0.29 & 20.76 & 1 & 13.33 & 0.16 & 0.19 & 5.08 & $5.01 \mathrm{E}-16$ \\
2 & 0.58 & 16.05 & 0.42 & 17.81 & 0.29 & 0.28 & 9.67 & $6.11 \mathrm{E}-19$ \\
3 & 0.59 & 12.68 & 0.38 & 15.68 & 0.39 & 0.26 & 8.62 & $1.33 \mathrm{E}-13$ \\
4 & 0.81 & 107.16 & 2.96 & 22.8 & 0.47 & 0.25 & 11.53 & $1.47 \mathrm{E}-14$ \\
5 & 0.81 & 14.56 & 0.34 & 20.71 & 0.57 & 0.24 & 12.09 & 0 \\
6 & 0.94 & 36.08 & 0.94 & 14.57 & 0.01 & 0.47 & 13.01 & $8.37 \mathrm{E}-16$ \\
the mean & 0.67 & 34.55 & 1.01 & 17.48 & 0.32 & 0.28 & 10 & $2.49 \mathrm{E}-14$ \\
$\mathrm{~S}$ & 0.23 & 36.56 & 1 & 3.68 & 0.21 & 0.1 & 2.9 & $5.34 \mathrm{E}-14$ \\
$\mathrm{~N}$ & 48 & 455 & 401 & 18 & 176 & 47 & 34 & 1870
\end{tabular}

It can be seen from Table 2 that the reasonable number of points determined by using the three parameters $i_{c}, i_{0}$, and $\beta$ of the Horton formula are 48, 455, 401 , and the minimum number of reasonable points obtained by three parameters is taken as a reasonable number of the points. So the number of reasonable points is determined by the parameters $i_{c}$; The reasonable number of the points determined by the Philip formula parameters $\mathrm{A}$ and $\mathrm{S}$ are 34, 1870 respectively, and the reasonable number of the points should be 34 ; the number of reasonable points obtained by using K-1 formula parameters $k, a, f_{0}$ is $18,176,47$ respectively, the number of reasonable points should be 18 . A comparison of the three models shows that the number of reasonable points determined by the parameter $\mathrm{K}$ in the $\mathrm{K}-\mathrm{l}$ model is the least, 18 . Taking the time nodes $2,5,10,15, \ldots, 90$ min, the corresponding values for the six sets of infiltration data of the actual test are interpolated by the quadratic parabolic interpolation method, and the average infiltration amount of the corresponding time nodes, the mean squared difference $\mathrm{S}$, coefficient of variation $\mathrm{CV}$ can be obtained. Then, the corresponding reasonable number of points $\mathrm{N}$ is calculated by the formula (3), and the figure of the mean square error, the average value, the reasonable number of points, and the coefficient of variation of the corresponding time nodes can be obtained, as shown in Figure 3.
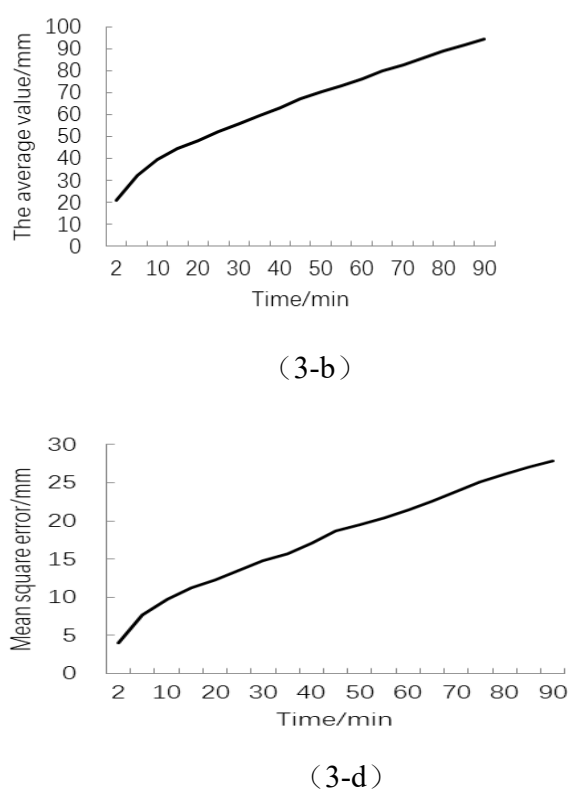

Figure 3. The variation of the mean square deviation, mean value, rational number of measured points 
It can be seen that the mean square error, the average value, the reasonable number of points, and the coefficient of variation obtained by the quadratic parabolic interpolation method increase with time. The mean square error, mean value, reasonable number of points, and coefficient of variation of the Kostiakov-lewis model are consistent with the change of the quadratic parabolic interpolation method, and the K-1 model is more reasonable.

\section{Conclusion}

(1) For the relationship between cumulative infiltration water and time, the K-l model has the best fitting effect, its R2 value is closer to 1 than the other two models.

(2) For the measured data in this paper, the number of reasonable points determined by the parameter $\mathrm{K}$ in the $\mathrm{K}-\mathrm{l}$ infiltration model is the least. It is indicated that the parameter $\mathrm{K}$ in the K-1 infiltration model should be used to determine the reasonable number of points in the soil infiltration test.

\section{Acknowledgments.}

The work is supported by the National Natural Science Foundation of China (51779174), the Tianjin University Students Innovation and the Entrepreneurship Project (201810061024) and Tianjin Agricultural Science and Technology Achievements Transformation and Promotion Project (201701150).

\section{References}

1. Wei,J.J., Yang,X.K., Zhang,Z.Q., (2013) Tian Haiyan. Difference of yield characteristics between different winter wheat lines under drip irrigation conditions. J. Northwest Journal of Agriculture., 22(4):44-48.

2. Jang,N., Shao,M.A., Lei,T.W., (2005) Study on the spatial variation of soil infiltration in the small watershed of the Loess Plateau. J. Journal of Soil and Water Conservation., 19(1):14-17.

3. Lei,Z.D., Yang,S.X., Xie,S.C., (1988) Hydrodynamics. M. Tsinghua University Press, Beijing.

4. Zhu,L.J., Zhang,G.H., Ren,Z.P., (2012) 4Comparison of methods for determination of soil infiltration. J. Soil and water conservation circular, 320:163-168. 